\title{
A structural approach to the design of FXR ligands
}

\author{
Dannielle Kydd-Sinclair ${ }^{1}$, Kimberly Watson ${ }^{1}$ \\ ${ }^{1}$ School Of Biological Sciences, University Of Reading, Reading, United Kingdom \\ E-mail: d.kydd-sinclair@pgr.reading.ac.uk
}

Over the last decade, the Farnesoid X Receptor (FXR) has emerged as a highly promising therapeutic target. The nuclear receptor is predominantly expressed in the liver, intestines, kidneys, adrenal glands and adipose tissues where it has been identified as the modulator of bile acid's signalling actions. Both free and conjugated bile acids can activate FXR, inducing its pleiotropic target genes which include those involved in bile acid homeostasis and lipid, glucose and cholesterol metabolism. FXR, like other classic nuclear receptors, comprises a ligand binding domain (LBD) connected to a DNA binding domain (DBD) via a hinge region. The 12 a-helix LBD harbours a highly flexible, hydrophobic ligand binding cavity, which once bound, causes a change in structural conformation whereby corepressor complexes are released and coactivator complexes are recruited, activating the transcription of target genes. Several synthetic and semi-synthetic agonists have already been described for FXR and co-crystal structures have shown that the architecture of the ligand scaffold can have a significant impact on the specific helices involved in interactions, thus the conformation that the receptor can adopt and therefore the activation of the receptor. Efforts into the design and development of FXR agonists has escalated due to their potential benefits in treating high impact pathologies and complications such as obesity, diabetes, atherogenesis and fatty liver diseases.

The aim of our work is to use molecular docking and three-dimensional quantitative structure activity relationships (3DQSAR) to obtain insight into how modifications and additions of structural elements to the bile-acid backbone can effect FXR activation, in an attempt to develop novel bile acid analogue ligands of FXR with improved potency. To date, we have used in silico docking to place novel compounds into the FXR ligand binding pocket to identify potential interactions that will be exploited and the respective helices that will be involved in ligand-induced binding. Work has focussed also on the expression of the FXR LBD and once the comparative analysis of the ligand compounds has taken place, co-crystallisation of the LBD with the lead compound will allow us to further understand how the ligand sits in the binding pocket, what important interactions are made and how the ligand can be altered to achieve maximal receptor activation. Proposed work by our group also includes determination of whether the DBD can be expressed. Optimisation of expression methods, currently underway, may allow us to express, purify and eventually solve the previously undescribed, structure of the FXR DBD together with the LBD.

Keywords: structure based drug design, farnesoid $\mathrm{X}$ receptor 